\title{
Demi-Disk Travelling Wave Accelerating Structure *
}

\author{
J. Gao \\ Laboratoire de L'Accélérateur Linéaire, IN2P3-CNRS \\ et Université de Paris-Sud, 91405 Orsay cedex, France
}

\begin{abstract}
The dark current in a normal disk-loaded accelerating structure is one of the main obstacles which limit the accelerating gradient going higher. In this paper a demi-disk accelerating structure is proposed in order to reduce the dark current. Its transverse rf focusing forces can reduce the effects of wakefields on the beam's emittance growth which is preferable in future linear colliders. 3D program PRIAM [1] has been used to calculate the field distributions and the dispersion curves of this structure. Estimations of the trajectories of the field emission electrons have been done.
\end{abstract}

\section{INTRODUCTION}

The next generation $\mathrm{TeV}^{+} e^{-}$linear colliders require that the accelerating structures support high accelerating gradient in order to keep the machine within a reasonable scale. The fact that various beam property control methols, such as BNS damping [2], energy spread compensation [3] and fundamental mode detuning [4], lose acceleration efficience emphasize also the importance of this requirement. It is found, however, that in a conventional axisymmetric disk-loaded accelerating structure, when the field level reaches certain value, appreciable electrons are emitted from the surface of the accelerating structure and some of them are captured by the travelling wave and accelerated continuously along the axis of the accelerating structure [5][6]. This captured electrons' flow is so-called dark current. The dark current has very wide energy spectrum and therefore produces lot of noises within detectors. On the other hand the dark current excites wakefields and vakes precious of power in the form of beam loading.

In this paper a demi-disk travelling wave accelerating structure is proposed aiming to reduce the dark current. In section 2 we will first look at the dark current in the case of conventional disk-loaded accelerating structures. In section 3 the demi-disk structure is investigated.

\section{CONVENTIONAL DISK-LOADED ACCELERATING STRUCTURE}

In order to get some general ideas of the behaviour of the field cmission electron in a conventional axisymmetric structure let's look at the longitudinal and the transverse motions of it.

* Part of this work was first presented at the LC92 linear collider workshop, Garmitsch, Germany, July 25 - August 2, 1992.
The longitudinal electric field inside an axisymmetric disk-loaded structure is expressed as:

$$
E_{z}(r, z, t)=E_{z}(r, z) \sin \left(\omega t-k_{g} z+\phi_{0}\right)
$$

where $\omega=2 \pi f, k_{g}=2 \pi / \lambda_{g}$ is the fundamental wave number of this travelling wave structure and $\phi_{0}$ is the initial emission phase of the field emission electron when it is emitted at $z=0$ and $t=0$. If only linear term is kept, the electric field near the axis can be expressed as:

$$
E_{z}(r, z, t)=E_{z}(0, z) \sin \left(\omega t-k_{g} z+\phi_{0}\right)
$$

In the following analytical treatment, $E_{z}(0, z)$ has been chosen to be a constant $E_{z 0}$. From eq. 2 we have

$$
\frac{d \gamma}{d z}=\frac{q E_{z 0}}{m_{0} c^{2}} \sin (\phi)
$$

where

$$
\phi=\omega t-k_{g} z+\phi_{0}=k \int_{0}^{z}\left(\frac{\gamma}{\left(\gamma^{2}-1\right)^{1 / 2}}-1\right) d z+\phi_{0}
$$

where $\gamma$ is the ratio between the electron's relativistic energy and the rest energy $m_{0} c^{2}, k=2 \pi / \lambda$ and $\lambda$ is the electronagnetic wavelength in free space. In eq. $4 k_{g}$ has been chosen equal to $k$ (the phase velocity of this travelling wave $\beta_{p}=\omega / k c=1$ through out this paper). When $\gamma \gg 1, \phi$ will be frozen at its asymptotic value [7]

$$
\begin{gathered}
\phi_{f}=\frac{1}{\alpha \sin \left(\phi_{0}+\delta \phi\right)}+\phi_{0} \\
\alpha=\frac{q E_{z 0}}{m_{0} c^{2} k}
\end{gathered}
$$

where $\delta \phi$ can be calculated from an empirical formula which is given as:

$$
\delta \phi(\text { degree })=19 E_{z 0}^{-0.9}
$$

where $E_{z 0}$ is in $\mathrm{MV} / \mathrm{cm}$. In fact it is found that $\delta \phi$ can be neglected if $\phi_{0}>60^{\circ}$. From eq. 3 and eq. 5 one can get the approximate expression of the final energy gain of longitudinally captured electrons with respect to the frozen phase and electron's longitudinal position $\mathrm{z}$ :

$$
\Gamma_{f}=1+\alpha \sin \left(\phi_{J}\right) k z
$$

In the following we will discuss the transverse motion of the field emission electron keeping the simpification of only using linear of field terms. It is well known that the 
transverse rf force acting on a particle with electric charge $q$ travelling at velocity $v_{z}$ is expressed as:

$$
F_{r}=\frac{q r k}{2}\left(1-\beta_{p} \beta_{z}\right) E_{z 0} \cos \left(\omega t-k z+\phi_{0}\right)
$$

where $r$ is the transverse deviation from the axis and $\beta_{z}=v_{z} / c$. Assuming that an electron is emilted at $t=0$ and $z=0$ with $\beta_{z}=0$ from the cavity wall, one can see from eq. 9 that the electron will be either focused or defocused depending on their initial emission phase $\phi_{0}$. When the field emission electrons's velocity approache that of light they will no longer feel the transverse rf force and some of them can be continuously accelerated near the axis along the structure as dark current if they are properly focused. Numerical simulation shows that at $\mathrm{S}$-band $(3 \mathrm{GHz})$ when $E_{z 0}=30 \mathrm{MV} / \mathrm{m}$ the field emitted electrons in a $60^{\circ}$ phase interval centered around the of peak $\left(\phi_{0}=90^{\circ}\right)$ are captured and accelerated to the end of the accelerating structure [8]. It is obvious that this large capture phase interval should be reduced if one want to increase accelerating gradient without raising the working frequency.

\section{DEMI-DISK TRAVELLING WAVE ACCELERATING STRUCTURE}

\section{A rf properties}

The proposed demi-disk accelerating structure is shown in Fig. 1. To compare with the SLAC type $2 \pi / 3$ mode structure, the same mode has bcen chosen for this demidisk structure. The standing wave electric field pattern of $2 \pi / 3$ mode is shown in Fig. 2. The dispersion curves are shown in Fig. 3 where the radius of the iris has been changed. It is interesting to notice that the group velocity at $\pi$ mode is not zero, and this is due to the fact that the structure has "quasi" $D / 2$ periodicity. Fig. 4 shows the dependences of the group velocity $v_{g}$ and the shunt impedance $R$ on the iris radius $r_{0}$. What should be pointed out are that the demi-disk structure has very large group velocity when the iris radius is small, and that when the iris radius is larger than some value the rf source might excite another mode which has the same resonant frequency as the desired working mode.

\section{$B$ beam dynamics}

The mution of the electron moving inside this structure can be found out from the rf force near the axis just like

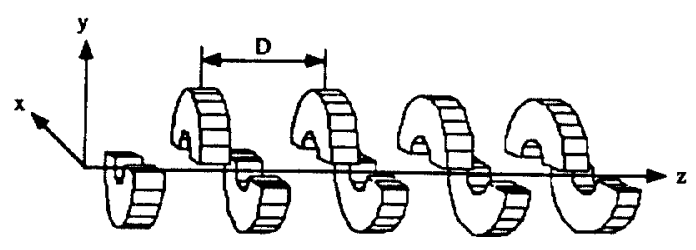

Figure 1: Demi-disk travelling wave structure

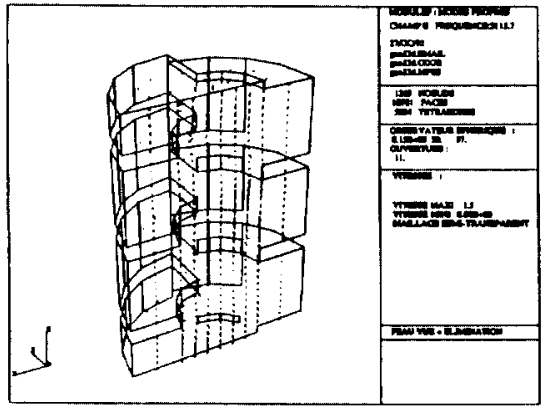

Figure 2: Electric field pattern of $2 \pi / 3$ mode

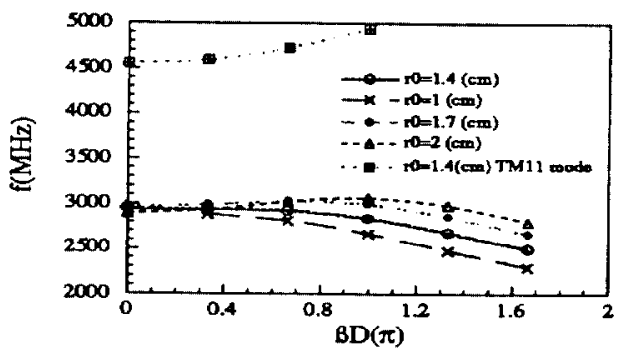

Figure 3: Dispersion curves

what has been done in the previous section. The longitudinal motion can be described by the same formulae derived for an axisymmetric structure. Now, we first look at the transverse motion of the electron in the vertical plane (symmetric plane, $\mathrm{x}=0$ ). According to the numerical results of PRIAM we know that $E_{z}, E_{x}$ and $H_{y}$ can be expressed as:

$$
\begin{array}{r}
\left.E_{z}(x, y, z, t)\right|_{x=0, y=0}=E_{z 0} \sin \left(\omega t-k z+\phi_{0}\right) \\
\left.E_{x}(x, y, z, t)\right|_{y=0}=x K_{e} \cos \left(\omega t-k z+\phi_{0}\right) \\
\left.E_{y}(x, y, z, t)\right|_{x=0, y=0}=E_{y 0} \cos \left(\omega t-2 k z+\phi_{0}\right) \\
\left.H_{y}(x, y, z, t)\right|_{y=0}=-x K_{h} \sin \left(\omega t-k z+\phi_{0}\right)
\end{array}
$$

From $\nabla \cdot E=0$

$$
\frac{\partial E_{x}}{\partial \boldsymbol{x}}+\frac{\partial E_{y}}{\partial y}+\frac{\partial E_{z}}{\partial z}=0
$$

we get

$$
\begin{gathered}
\left.E_{y}(x, y, z, t)\right|_{x=0}=E_{y}(0,0, z, t)-\int_{0}^{y}\left(\frac{\partial E_{x}}{\partial x}+\frac{\partial E_{z}}{\partial z}\right) d y \\
=E_{y 0} \cos \left(\omega t-2 k z+\phi_{0}\right)-y K_{e} \cos \left(\omega t-k z+\phi_{0}\right) \\
\quad+y k E_{z 0} \cos \left(\omega t-k z+\phi_{0}\right)
\end{gathered}
$$

Figure 4: $v_{g} / c$ and $R$ vs $r_{0}$ 
From $\nabla \times H=\epsilon_{0} \frac{\partial E}{\partial t}$, we have

$$
\begin{gathered}
\frac{\partial H_{x}}{\partial y}=\left(\frac{\partial H_{y}}{\partial x}-\epsilon_{0} \frac{\partial E_{z}}{\partial t}\right) \\
I_{x}=\int_{0}^{y}\left(\frac{\partial H_{y}}{\partial x}-\epsilon_{0} \frac{\partial E_{z}}{\partial t}\right) d y \\
=-y K_{h} \sin \left(\omega t-k z+\phi_{0}\right) \\
-y \epsilon_{0} \omega E_{z 0} \cos \left(\omega t-k z+\phi_{0}\right)
\end{gathered}
$$

where the constants $E_{y 0}, H_{y 0}, K_{e}$ and $K_{h}$ can be determined from the numerical results of PRIAM. We know from eq. 15 and eq. 17 that the transverse if force acting on a particle can be expressed as:

$$
\begin{gathered}
F_{y}=q E_{y 0} \cos \left(\omega t-2 k z+\phi_{0}\right) \\
+q y k\left(1-\beta_{p} \beta_{z}\right) E_{z 0} \cos \left(\omega t-k z+\phi_{0}\right) \\
-q y K_{e} \cos \left(\omega t-k z+\phi_{0}\right)-q y \mu_{0} v_{z} K_{h} \sin \left(\omega t-k z+\phi_{0}\right)
\end{gathered}
$$

As for the expression of the rf force in the horizontal plane $(y=0)$, similarly, we have

$$
F_{x}=q x K_{e} \cos \left(\omega t-k z+\phi_{0}\right)+q x \mu_{0} v_{z} K_{h} \sin \left(\omega t-k z+\phi_{0}\right)
$$

For the accelerated electrons $\left(\gamma \gg 1\right.$ and $\left.\beta_{z} \approx 1\right)$, by ignoring the first term in eq. 18 which doesn't synchronize with the electrons and using eq. 5 , the transverse rf forces can be rewritten as:

$$
\begin{gathered}
F_{y}=-q y K_{e} \cos \left(\phi_{f}\right)-q y \mu_{0} v_{z} K_{h} \sin \left(\phi_{f}\right) \\
F_{x}=q x K_{e} \cos \left(\phi_{f}\right)+q x \mu_{0} v_{z} K_{h} \sin \left(\phi_{f}\right)
\end{gathered}
$$

It is obvious that if in one plane it is focusing in another plane it will be defocusing. In order to have focusing for accelerated beam in both transverse planes, rotating the polarization of the demi-disks along the structure is necessary as shown in Fig. 5. The philosophy of this design is to deflect the low energy field emission electrons in each short uniform section as shown in Fig. 1, while keep focusing the accelerated beam in the whole accelerating structure against the transverse wakefields. In this paper attentions will be put to the motions of the field emission electrons in the uniform section only. Based on the linear of force expressions in eq. 18 and eq. 19, field emission electrons' trajectories have been calculated and shown in Fig. 6 and Fig. 7. From these two figures it can be seen clearly that few electrons can be captured as dark current since in one transverse plane field emission electron is focused in another plane it will be defocused.

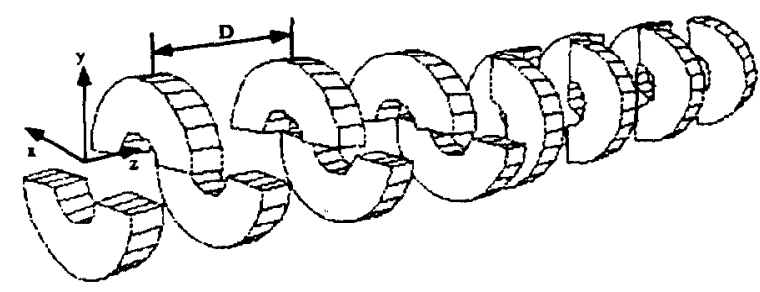

Figure 5: Demi-disk structure with rotating disks

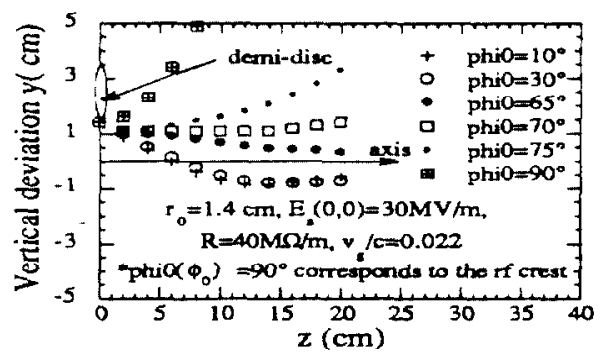

Figure 6: The trajectories of the field emission electrons in the vertical plane with different emission phase $\phi_{0}$

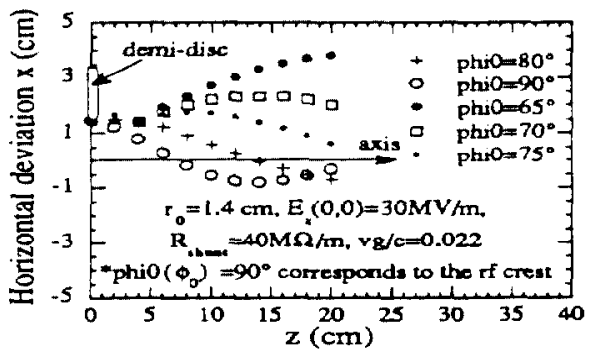

Figure 7: The trajectories of the field emission electrons in the horizontal plane with different emission phase $\phi_{0}$

\section{DISCUSSIONS}

Even the demi-disk structure discussed above is proposed to reduce the dark current, it might have other applications such as of wigglers and the positron generation linacs. What should be pointed out is that if it is used as a positron generation linac, the adjacent demi-disks should have $90^{\circ}$ polarization difference in order to focus quickly the low energy positrons in both transverse planes.

\section{ACKNOWLEDGEMENTS}

The author appreciates the helps of Y. Thiery, G. Le Meur and F. Touz during using PRIAM. He appreciates also the discussions with G. Bienvenu, P. Brunet, J. Le Duff, T. Garvey and A. Dovbnia.

\section{REFERENCES}

[1] G. Le Meur and F. Touz, Proc. of EPAC, June 12-16, 1990, Nice, France, P. 1693.

[2] V. Balakin, A. Novokhasky and V. Smirnov, Proceedings of the workshop on "Physics of linear colliders", Capri, June 13-17, 1988, p. 167.

[3] R. D. Ruth, SLAC-PUB-4541, 1988.

[4] J. Gao, "Fundamental mode detuning travelling wave accelerating structure", presented at LC92 linear collider workshop, Garmitsch, Germany, July 25 - August 2, 1992, in these proceedings.

[5] G. Bienvenu, Proceedings of EPAC, June 7-11, 1988, Rome, Italy, P. 970.

[6] G. Bienvenu and P. Brunet, Proceedings of EPAC, June 12-16, 1990, Nice, France, P. 934.

[7] J. Gao, Proceedings of EPAC, Berlin, March 24 28, 1992, p. 584 .

[8] G. Bienvenu, P. Fernandes and R. Parodi, Nucl. Instr. and Methods, A320(1992) pp. 1-8. 